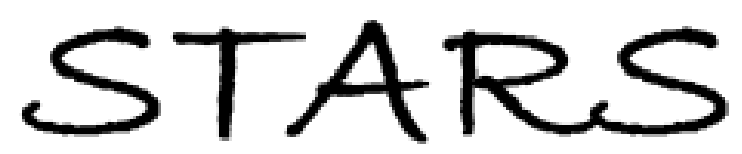

University of Central Florida

STARS

$1-1-2002$

\title{
Inhomogeneous nanoscale polymer-dispersed liquid crystals with gradient refractive index
}

Hongwen Ren

University of Central Florida

Shin-Tson Wu

University of Central Florida

Find similar works at: https://stars.library.ucf.edu/facultybib2000

University of Central Florida Libraries http://library.ucf.edu

This Article is brought to you for free and open access by the Faculty Bibliography at STARS. It has been accepted for inclusion in Faculty Bibliography 2000s by an authorized administrator of STARS. For more information, please contactSTARS@ucf.edu.

\section{Recommended Citation}

Ren, Hongwen and Wu, Shin-Tson, "Inhomogeneous nanoscale polymer-dispersed liquid crystals with gradient refractive index" (2002). Faculty Bibliography 2000s. 3426.

https://stars.library.ucf.edu/facultybib2000/3426

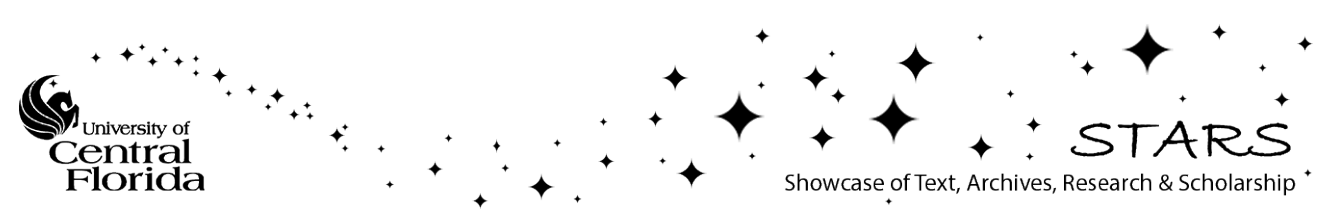




\section{Inhomogeneous nanoscale polymer- dispersed liquid crystals with gradient refractive index}

Cite as: Appl. Phys. Lett. 81, 3537 (2002); https://doi.org/10.1063/1.1519102

Submitted: 12 July 2002 . Accepted: 13 September 2002 . Published Online: 28 October 2002

Hongwen Ren, and Shin-Tson Wu

\section{ARTICLES YOU MAY BE INTERESTED IN}

Tunable Fresnel lens using nanoscale polymer-dispersed liquid crystals Applied Physics Letters 83, 1515 (2003); https://doi.org/10.1063/1.1604943

Tunable electronic lens using a gradient polymer network liquid crystal Applied Physics Letters 82, 22 (2003); https://doi.org/10.1063/1.1534915

Polarizer-free and fast response microlens arrays using polymer-stabilized blue phase liquid crystals

Applied Physics Letters 96, 113505 (2010); https://doi.org/10.1063/1.3360860

\section{Applied Physics Letters}

Mid-IR and THz frequency combs special collection

\section{Read Now!}




\title{
Inhomogeneous nanoscale polymer-dispersed liquid crystals with gradient refractive index
}

\author{
Hongwen Ren and Shin-Tson $\mathrm{Wu}^{\mathrm{a})}$ \\ School of Optics/CREOL, University of Central Florida, Orlando, Florida 32816
}

(Received 12 July 2002; accepted 13 September 2002)

\begin{abstract}
Inhomogeneous polymer-dispersed liquid crystal devices having gradient droplet distribution were fabricated and their phase retardation characterized. The gradient refractive index profile can be used as switchable prism gratings, and positive and negative lenses with tunable focal lengths. Such a tunable electronic lens is a broadband device and can be used for unpolarized light. (C) 2002 American Institute of Physics. [DOI: 10.1063/1.1519102]
\end{abstract}

Polymer-dispersed liquid crystal (PDLC) is a composite material consisting of liquid crystal (LC) droplets dispersed in a polymer matrix. Two types of PDLC devices have been developed and used for displays ${ }^{1,2}$ and tunable wavelength filters. $^{3-5}$ For display applications, the LC droplet size is around $1 \mu \mathrm{m}$ so that light scattering dominates in the visible region. For telecom light switches, the PDLC droplets are in nanoscale so that the polymer matrix is optically isotropic with respect to the incident light. Holographic PDLC is such an example. ${ }^{6,7}$ In the voltage-off state, no light scattering occurs. In a high-voltage state, the nano LC droplets are reoriented by the electric field resulting in a phase change. Holographic PDLC has been used as a switchable Bragg grating with $\sim 100 \mu \mathrm{s}$ response time. In these PDLC devices, the droplet sizes are intended to be homogeneous although a small variation is unavoidable. The electro-optic properties of these PDLC films are presumably uniform across the whole device.

In this letter, we demonstrate a third type PDLC device that has inhomogeneous droplet size distribution. In such PDLC, the inhomogeneous droplet size distribution is obtained by exposing the LC/monomer with a uniform ultraviolet (UV) light through a patterned photomask. For example, if the photomask has a linear transmission gradient across the UV exposure area, then the formed PDLC film would exhibit a gradient refractive index profile. The area exposed to a weaker UV intensity would produce a larger droplet size. These larger LC droplets would exhibit a larger phase retardation than that of smaller droplets. By varying the photomask patterns, various optical devices, e.g., variable focus positive and negative lenses, tunable prisms and grating, and microlens arrays can be realized. ${ }^{8}$

We used the polymerization-induced phase separation ${ }^{9}$ method to prepare the inhomogeneous PDLC. Our photomask is chosen with continuously variable neutral transmission, as shown in Fig. 1. When the uniform UV light passes through the photomask, the intensity varies almost linearly across the sample. The stronger UV accelerates the phase separation between the curable monomer and LC resulting in a smaller droplet size. In contrast, the weaker UV exposure causes a slower phase separation which leads to larger LC

a)Electronic mail: swu@mail.ucf.edu droplets. As shown in Fig. 1, PDLC with gradient droplet size distribution is produced.

The PDLC formed by the photopolymerization-induced phase separation method usually shows weak anchoring. Under such a circumstance, the PDLC driving voltage $V_{d}$ (the critical voltage) is dependent on the droplet size $(D)$ as: ${ }^{10}$

$$
V_{d}=C / \sqrt{D}
$$

where $C$ is a constant. From Eq. (1), a larger droplet would have a lower operating voltage. Conversely, a smaller droplet would require a higher voltage. When a uniform voltage is applied to a linearly varying PDLC cell as depicted in Fig. 1, the LC directors within a droplet have a different degree of reorientation, according to Eq. (1). As a result, the induced spatial refractive index change should have a parabolic profile. This approach is different from the LC-based optical phased arrays where the phase profile is digitally synthesized by varying the voltage of each pixel. ${ }^{11}$ An outstanding feature of our approach is the use of a uniform voltage to generate gradient phase profile. As a result, the electrode design is very simple.

To prepare PDLC cells, the UV-curable monomer NOA-81 (Norland) and E7 LC (Merck, $\Delta n=0.225, \Delta \varepsilon$ $=14.5$ ) were mixed at $70: 30 \mathrm{wt} \%$ ratio. The mixture was then injected into an empty glass cell with inner surfaces coated with indium-tin-oxide layers. The cell gap was measured to be $d=11.2 \mu \mathrm{m}$. To prove the principle, a continuously variable density filter (A41-960, Edmund Industrial Optics) was used as the photomask. The optical density varies linearly (from 0.04 to 2.00) from one edge to another (5 $\mathrm{cm}$ long). In our experiments, the UV (from Loctite) exposure intensity was $350 \mathrm{~mW} / \mathrm{cm}^{2}$, cure temperature $\sim 50{ }^{\circ} \mathrm{C}$ and curing time $300 \mathrm{~s}$.

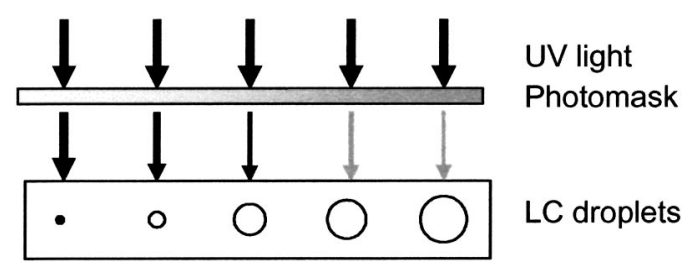

FIG. 1. Fabrication of an inhomogeneous PDLC using a patterned photomask. 


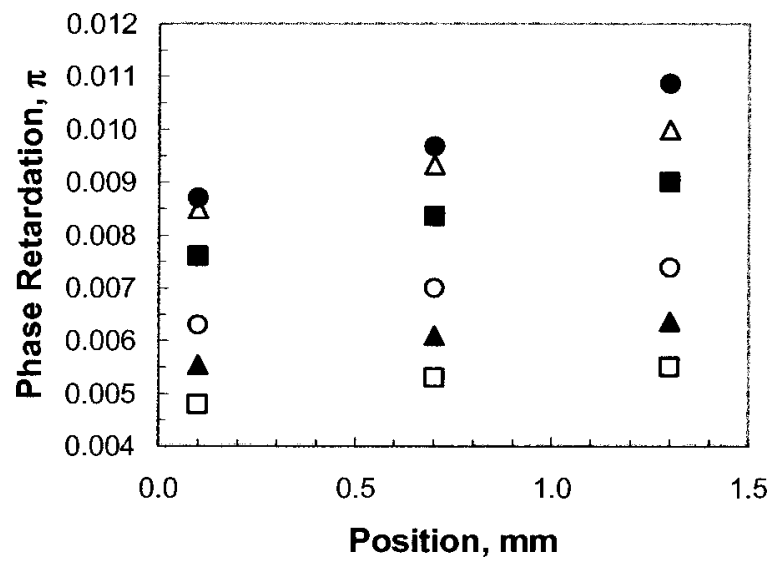

FIG. 2. The measured phase retardation of an inhomogeneous PDLC device. Nolan NOA81: E7 $=70: 30$. Cell gap $d=11.2 \mu \mathrm{m}, \lambda=633 \mathrm{~nm}$. The three spots chosen for measurements were $0.6 \mathrm{~mm}$ apart. From top to bottom, the applied voltage is $V=0,60,80,100,110$, and $125 \mathrm{~V}_{\mathrm{rms}}$, respectively.

The prepared PDLC sample is clear at the edge with strong UV exposure indicating that the droplet sizes are smaller than the visible wavelength $(D<\lambda)$. The weaker exposure edge appears a little bluish which implies that the formed LC droplet size is comparable to a blue wavelength $(\lambda \sim 400 \mathrm{~nm})$. The entire sample is highly transparent at the $\mathrm{He}-\mathrm{Ne}$ laser wavelength $(\lambda=633 \mathrm{~nm})$ which we used for measuring phase retardation.

The phase separation mechanism of the inhomogeneous PDLC is somewhat different from that of a uniform-sized PDLC. In the inhomogeneous PDLC, the photopolymerization takes place preferentially in the high UV intensity regions. The consumption of monomers in these regions lowers their chemical potential. This pushes the monomers to diffuse from the low to high intensity region. On the contrary, the LC molecules diffuse from the high to low intensity direction to balance the chemical potential. As a result, in an inhomogeneous PDLC sample, not only droplet sizes but also their concentrations exhibit gradient distributions. The region having a larger droplet also has a higher LC concentration. Therefore, in the voltage-off state the inhomogeneous
PDLC sample is expected to exhibit gradient phase retardation.

To characterize the phase retardation $(\delta=2 \pi d \Delta n / \lambda)$ of the inhomogeneous PDLC cell, we chose three spots separated by $0.6 \mathrm{~mm}$. At each spot, we measured the voltagedependent transmittance using a $\mathrm{He}-\mathrm{Ne}$ laser under the crossed and parallel polarizer conditions. From the measured transmittances, phase retardation was calculated. ${ }^{12}$ Results are depicted in Fig. 2.

At $V=0$, a gradient phase retardation profile is indeed observed. The location with a larger droplet exhibits a higher phase retardation. In the voltage-off state, the LC directors within a droplet are almost randomly distributed. The average refractive index $\left.\left(n=n_{e}+2 n_{o}\right) / 3\right)$ of the LC droplets is larger than that of the polymer matrix $\left(n_{p} \sim n_{o}\right)$. The larger phase retardation observed in the larger droplet region is echoed by the higher LC concentration. As the voltage is gradually increased, the LC directors are reoriented toward the electric-field direction. In the very high-voltage regime, the refractive index is decreased from $n$ to $n_{o}$ and the phase retardation becomes flat across the whole sample, regardless of droplet sizes. Owing to the small droplet sizes, the required driving voltage is greater than $100 \mathrm{~V}_{\mathrm{rms}}$. To lower the operating voltage, the methods developed for holographic PDLC can be applied here as well. ${ }^{7}$

As shown in Fig. 2, the phase retardation of the $11 \mu \mathrm{m}$ thick inhomogeneous PDLC sample is relatively small. To increase phase retardation, a higher birefringence LC mixture or thicker cell gap can be considered. The tradeoff of a thicker cell is the increased operating voltage. Thus, high birefringence LC mixtures are preferred.

In some applications such as prism grating and tunable focus eyeglasses, a steep phase gradient is needed. To do so, we ought to compress the distance from the smallest to the largest droplets. For proving the principle, we demonstrated a prism grating using an inhomogeneous PDLC cell. A photomask with a periodic gradient light transmission was used. The sample fabrication processes are the same as mentioned before. Two samples were prepared: One was exposed at $I$ $=270 \mathrm{~mW} / \mathrm{cm}^{2}$ for $10 \mathrm{~s}$ and the second at $I=350 \mathrm{~mW} / \mathrm{cm}^{2}$

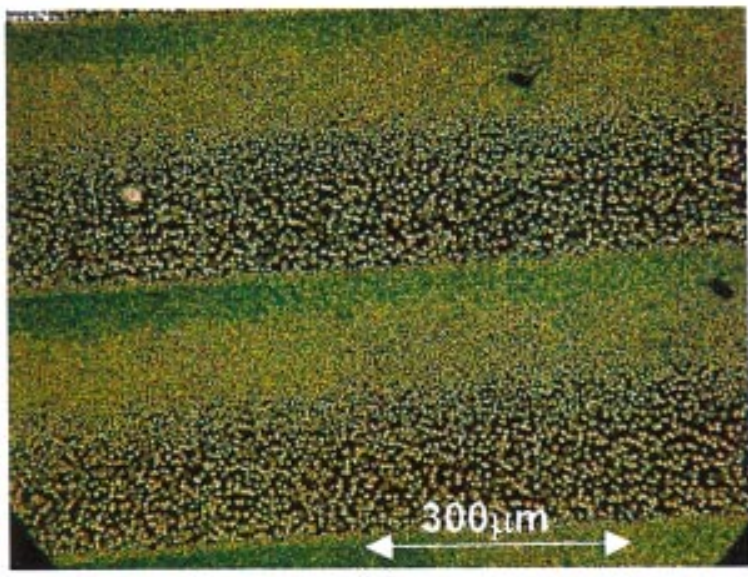

(a)

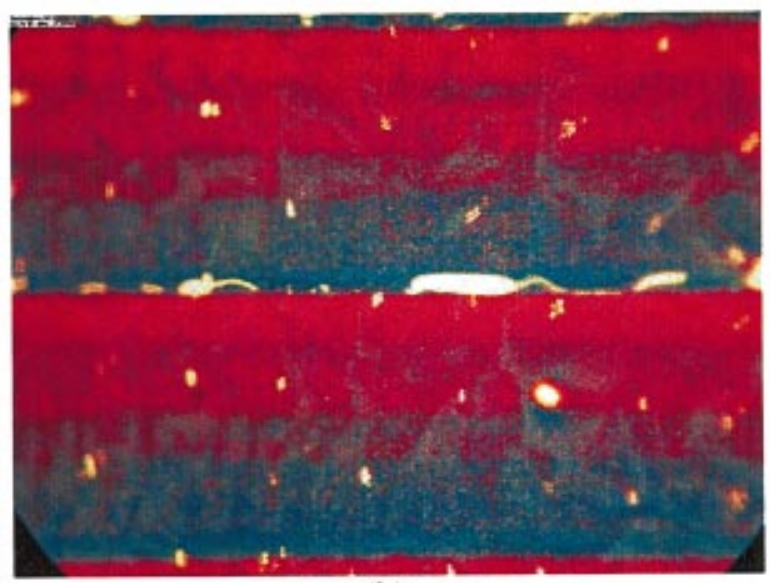

(b)

FIG. 3. (Color) Optical polarizing microscope photos of two PDLC samples with gradient droplet size distribution. LC composite: $70 \%$ NOA81 and $30 \%$ E7. (a) UV exposure intensity at $I=270 \mathrm{~mW} / \mathrm{cm}^{2}$ for $10 \mathrm{~s}$, and (b) $I=350 \mathrm{~mW} / \mathrm{cm}^{2}$ for $20 \mathrm{~s}$. 


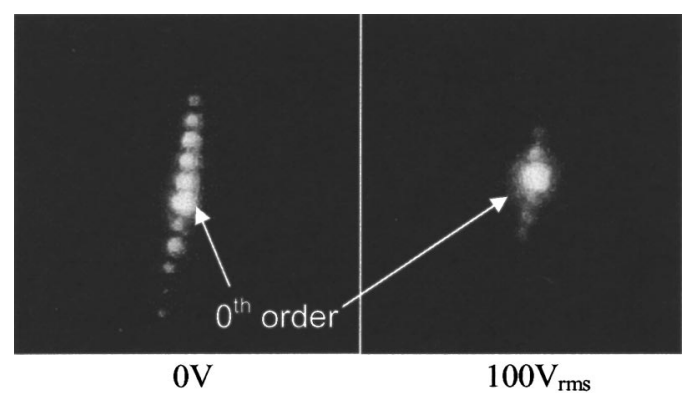

FIG. 4. Diffraction properties of a prism grating at $V=0$ (left) and $100 \mathrm{~V}_{\mathrm{rms}}$ (right). The inhomogeneous PDLC employed for this experiment is the one shown in Fig. 3(b). $\lambda=514 \mathrm{~nm}$.

for $20 \mathrm{~s}$. Their microscopic photos are shown in Figs. 3(a) and 3(b), respectively. It is clear that both samples have formed periodic prism grating structures. In Fig. 3(a), different LC droplet sizes are resolved within each period; while in Fig. 3(b), the LC droplets are too small to be resolved by microscope. However, it shows birefringence colors between crossed polarizers. In this report, we did not try to identify the droplet morphology by scanning electron microscope. Instead, we tested the diffraction properties of the second sample using an argon-ion laser $(\lambda=514 \mathrm{~nm})$. Results are shown in Fig. 4.

From Fig. 4, at $V=0$, the diffraction is asymmetric, i.e., more laser energy is diffracted to the positive than negative orders. This is the key feature of the prism grating. The noises appeared in the negative orders originate from the smooth edge of the periodic grating photomask we employed. If the grating edge is sharp, the negative diffraction orders should not occur. To erase the grating, we applied $\sim 100 \mathrm{~V}$ of ac voltage to the inhomogeneous PDLC cell. Since the grating is erased, the laser energy is concentrated in the zeroth order. The rise and decay times were measured to be $80-160 \mu$ s depending on the droplet sizes. This fast response indicates that the droplet sizes are truly nanoscale.

In addition to the prism grating, the inhomogeneous PDLC opens a new door for other applications. For instance, we could fabricate variable focus positive and negative lenses using photomasks with circular gradient transmissions as shown in Figs. 5(a) and 5(b), respectively. The expected droplet size distributions are sketched below the photomasks. At $V=0$, the $\mathrm{LC}$ directors inside the droplets are assumed to be randomly oriented. The area with a larger droplet size will exhibit a higher refractive index. Thus, Figs. 5(a) and 5(b) correspond to a positive and negative lens, respectively. As the voltage increases, the refractive index is decreased and
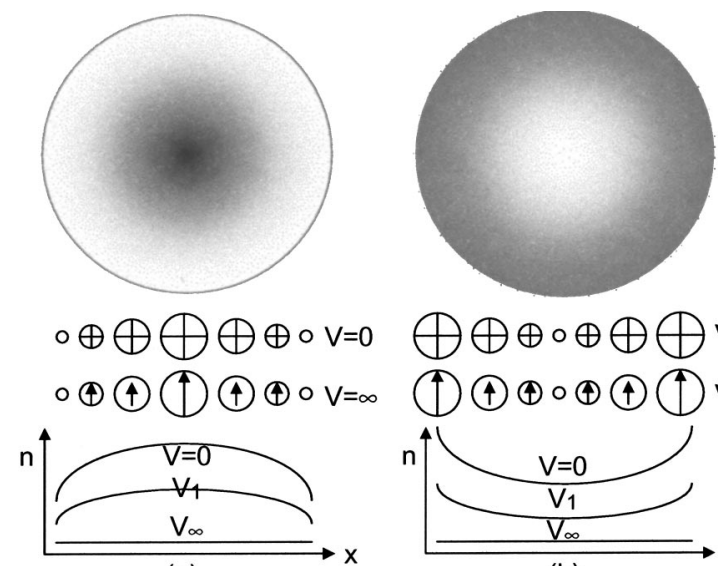

(a)

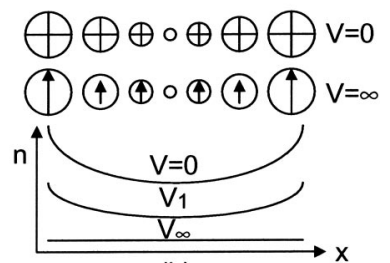

(b)

FIG. 5. Operating principles of a tunable (a) positive lens and (b) negative lens using the inhomogeneous PDLC devices. Top: Photomasks, middle: LC orientations at $V=0, V_{1}$ and $V=\infty$, bottom: The expected refractive index profiles.

the profile is flattened. Finally at $V=V_{\infty}$, the lens effect vanishes.

In conclusion, we have demonstrated a PDLC with gradient droplet size distribution. In the voltage-off state, the device exhibits a gradient phase profile. As the applied voltage increases, the phase profile is flattened and eventually vanished in a high-voltage regime. Such inhomogeneous PDLC can be used as prism grating and electronic lenses.

The authors would like to thank Y. B. Huang for providing the prism grating photomask. This work is supported by DARPA under Contract No. DAAD19-02-1-0208.

${ }^{1}$ J. L. Fergason, SID Tech. Digest 16, 68 (1985).

${ }^{2}$ J. W. Doane, A. Golemme, B. G. Wu, and S. Zumer, Appl. Phys. Lett. 48, 269 (1986).

${ }^{3}$ S. Matsumoto, M. Houlbert, T. Hayashi, and K. Kubodera, Appl. Phys. Lett. 69, 1044 (1996).

${ }^{4}$ S. Matsumoto, K. Hirabayashi, S. Sakata, and Takayoshi Hayashi, IEEE Photonics Technol. Lett. 11, 442 (1999).

${ }^{5}$ D. E. Lucchetta, R. Karapinar, A. Manni, and F. Simoni, J. Appl. Phys. 91, 6060 (2002).

${ }^{6}$ R. L. Sutherland, V. P. Tondiglia, and L. V. Natarajan, Appl. Phys. Lett. 64, 1074 (1994).

${ }^{7}$ T. J. Bunning, L. V. Natarajan, and V. P. Tondiglia, Annu. Rev. Mater. Sci. 30, 83 (2000).

${ }^{8}$ H. Ren and S. T. Wu, US patent pending (2002).

${ }^{9}$ J. L. West, Mol. Cryst. Liq. Cryst. 157, 427 (1988).

${ }^{10}$ S. T. Wu and D. K. Yang, Reflective Liquid Crystal Displays (Wiley, New York, 2001), Chap. 7.

${ }^{11}$ P. F. McManamon, T. A. Dorschner, D. L. Corkum, L. Friedman, D. S. Hobbs, M. Holz, S. Liberman, H. Q. Nguyen, D. P. Resler, R. C. Sharp, and E. A. Watson, Proc. IEEE 84, 268 (1996).

${ }^{12}$ S. T. Wu, U. Efron, and L. V. Hess, Appl. Phys. Lett. 44, 1033 (1984). 